\title{
Use of multi-domain robots in search and rescue operations - contributions of the ICARUS team to the euRathlon 2015 challenge
} \author{
Balta $^{8}$, Halil Beglerović ${ }^{8}$

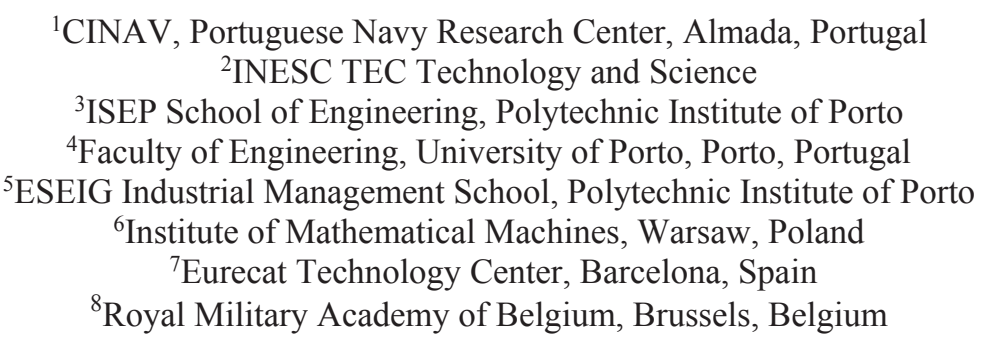

Mario Monteiro Marques ${ }^{1}$, Rui Parreira ${ }^{1}$, Victor Lobo ${ }^{1}$, Alfredo Martins ${ }^{23}$, Aníbal Matos ${ }^{24}{ }_{\text {Nuno }}$ Cruz $^{24}$, José Miguel Almeida ${ }^{23}$, José Carlos Alves ${ }^{24}$, Eduardo Silva ${ }^{23}$, Janusz Będkowski ${ }^{6}$, Karol Majek ${ }^{6}$, Michał Pełka $^{6}$, Paweł Musialik ${ }^{6}$, Hugo Ferreira ${ }^{25}$, André Dias ${ }^{23}$,Bruno Ferreira ${ }^{2}$, Guilherme Amaral ${ }^{2}$, André Figueiredo $^{24}$, Rui Almeida ${ }^{2}$, Filipe Silva ${ }^{2}$, Daniel Serrano ${ }^{7}$, German Moreno ${ }^{7}$, Geert De Cubber ${ }^{8}$, Haris

\begin{abstract}
Today, in our landscape perception exists a gap that needs to be fulfilled. It's important to increase the coverage, temporal and spatial resolution in order to cover this gap, as well as reduce costs with human resources that usually take this kind of tasks. Unmanned Autonomous vehicles with their inherent autonomy and reduced needs of human and communication resources, can provide additional capabilities and a new innovative solution to this problem

This paper presents and describes the participation of ICARUS Team at euRathlon 2015 and the importance of this type of events performed with multiple unnamed systems.
\end{abstract}

Keywords- Unmanned Vehicles; Search and Rescue, Interoperability

\section{INTRODUCTION}

EuRathlon 2015 is a competition, inspired by the 2011 Fukushima accident, evolving teams of land, sea and flying robots cooperating and working together to fulfil the defined objectives like collecting environmental data, survey the scene or identify critical hazards. The Enel-owned thermal power station at Torre del Sale will host a robotic competition involving all three domains: air, sea and land. The scene of a disaster will be created: cooperating robots will search for workers in ruined buildings, leaks of substances and damage to underwater structures and other emergency response tasks, all in a race against the clock [1].

Intelligent robots for disaster response are an urgent need all over the world in terms of robotics research, industry and emergency services. euRathlon provide not only a place and time to researchers meet and trade acknowledgments and experiences, as well as a platform to perform critical exercises and gaining experience in damage control and prevention in a cooperative way.

The ICARUS (Integrated Components for Assisted Rescue and Unmanned Search Operations) project is a large European research project with 24 partners from 10 European countries. It concentrates on the development of unmanned (USV, UAV, UGV) search and rescue technologies for detecting, locating and rescuing humans. All this systems need to share information between them and interoperability is an important issue to take in account [2].

ICARUS Team will bring to us in this event the LIFT VI, ROAZ, MARES, TEODOR and indoor oriented SUGV.

The remains of this paper are organized as follows: in Section II, we present the description of the scenarios. Section III, we describe the adaption strategy used in ICARUS vehicles. Section IV presents the air component. In section $\mathrm{V}$ we describe the sea component. In section VI is present the land component. Finally we present some conclusions.

\section{DESCRIPTION OF THE SCENARIOS}

The scenarios are defined in three different categories: The Grand Challenge; Sub-Challenge and Trial. 
The Grand Challenge consists of a three-domain scenario sea(S), land (L) and air (A) with three mission goals:

- Mission A: search for missing workers.

- Mission B: Reconnaissance and environmental survey of a building.

- Mission C: Pie inspection and stemming the leak.

To fully accomplish the Grand Challenge, the three missions should be completed within 100 minutes. As important as time limit, team strategy on how to use their robots played an important role on the mission success.

The Sub-Challenge is a two-domain scenario which are similar to the GC scenarios although with slight differences.

- Sub-Challenge $(\mathrm{L}+\mathrm{A})$ : survey the building and search for a missing worker.

- Sub-Challenge $(\mathrm{S}+\mathrm{A})$ : Pipe inspection and search for a worker.

- Sub-Challenge (L+S): Stem the leak.

Scoring in the Challenges take into account not only the time limit, but how well a robot performs in a specific task and how good a robot performs in terms of autonomy.

The Trials are divided in each domain in two land trials, two sea trials and two air trials.

\section{INTEROPERABILITY}

Interoperability may be defined as the capability of robots to operate in synergy for the execution of assigned tasks by sharing data, intelligence and resources. To ensure interoperability, ICARUS proposed the adaptation of the different platforms to a single standard interface [3]. The ICARUS standard interface then, acts as the glue among the different technologies of the ICARUS team [4]. It is based on the Joint Architecture for Unmanned Systems (JAUS) which is fairly aligned with the ICARUS purposes.

To be interoperable with the ICARUS team, a new system can directly integrate this interface. However, most robotics platforms currently are based on either proprietary or open-source middleware's. To integrate a new system then, an alternative is to implement an adapter to the robot-specific middleware. Figure 1 illustrates both cases: robots A and B are using their own middleware while robot $\mathrm{C}$ is natively integrated.

ICARUS developments are heavily based on the end-users feedback. According to end-users, SAR teams (search and rescue teams) are irremediably faced with a massive overload of work, so "sacrificing" people to operate the robotic tools is not an easy compromise. Therefore, a single Standard Command, Control and Intelligent (C2I) system was developed to operate the different systems in a mission.

The ICARUS project has validated the interoperability standard through a sequence of trials where the different collaboration between robots where tested at task and mission level. An initial integration of each unmanned platform and the C2I served as a

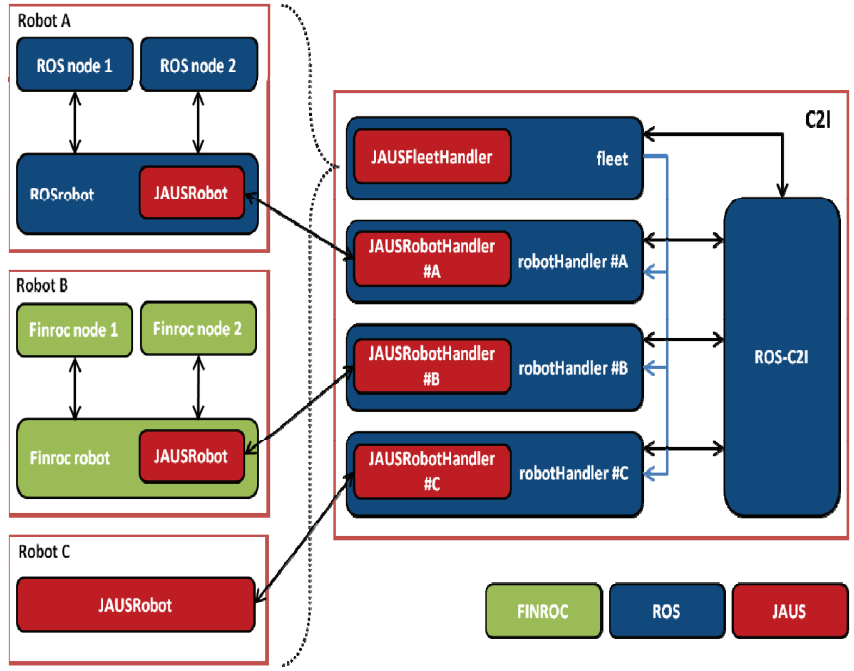

Figure 1 - ICARUS robots integration strategy

benchmark for verification of the ICARUS standard interface.

After corrective actions were applied, interoperability between ICARUS platforms was validated in real operations on a search and rescue scenarios.

For ICARUS, the euRathlon competition was a further opportunity to validate the developments of the project in a realistic scenario that was not specified and prepared by the project itself. EuRathlon also allowed demonstrating how easily a robot can be integrated in the ICARUS network. Two new robots were used by the ICARUS team. TEODOR, a large ground robot provided by RMA and MARES an underwater robot provided by INESC.

Thanks to the ICARUS interoperability, all the robots were able to provide live data during the operations. Given this, every operator was aware of the performance of all the robots as there was constant communication between domains. This fact made possible several achievements:

- The large ground robot was able to directly go to the unblocked entrance of the building. It was possible because the outdoor quadrotor detected quickly the unblocked entrance and communicated it to the land operator.

- The small ground robot and the underwater robot detected the correct valves of the leaking pipes. This operation was needed to be in synchronization. All operators communicated to the others the correct leaking pipe as soon as the robot had detected it.

- Every operator was aware of all the OPI's already detected, including missing workers, blocked and unblocked entrances and damages in the environment. 
- A cooperative mapping mission was done, fusing data from the ground and aerial vehicles [5].

The results from each domain were fused using the C2I and provided to the organizers for their evaluation. The following image shows the result from the Grand Challenge, with the multi-domain maps, the trajectory of the different robots, and the evidences of the automatically detected OPI's (objects of potential interest).

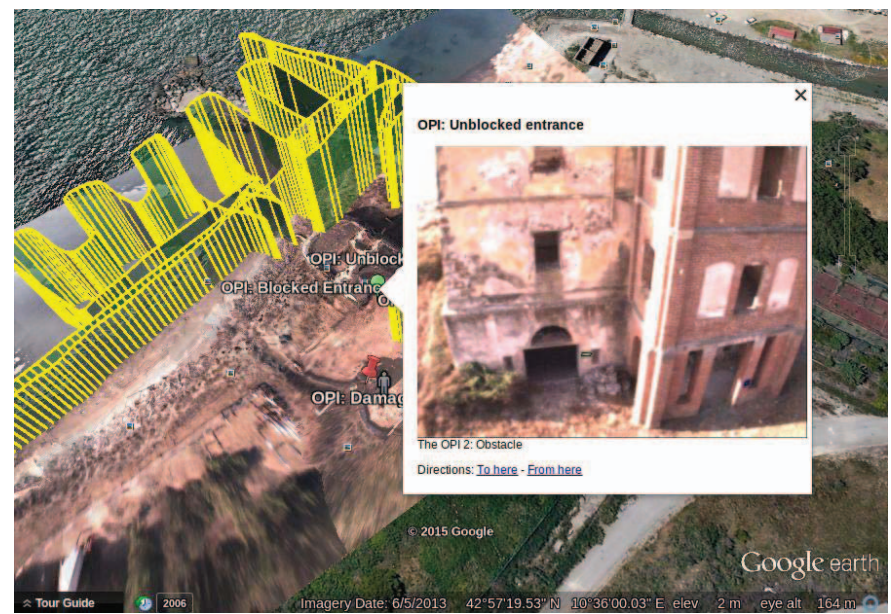

Figure 2 - Fused results from air and ground

3D data gathered by different platforms can be merged into a single 3D map of the environment, like Figure 2. The model created with this approach has advantages of both aerial (high coverage in short time) and ground (high accuracy) mapping. Figure 3 shows two maps as different layers, while Figure 4 shows the merged map. Algorithms used in merging process are described in [3].

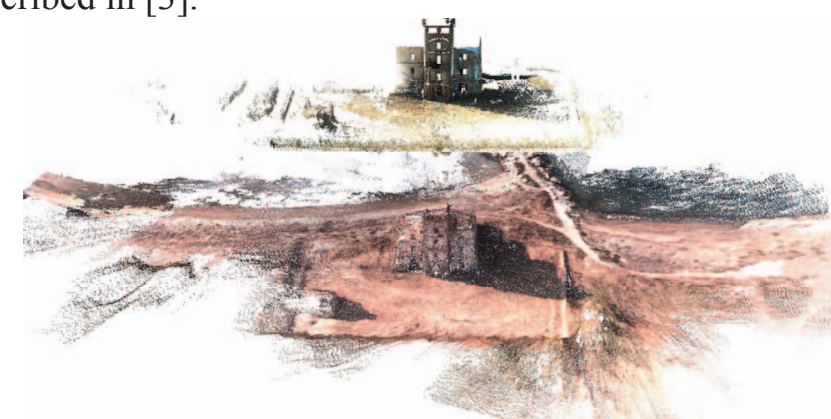

Figure 3 - Two layers of 3D map; Top - ground, bottom aerial data.

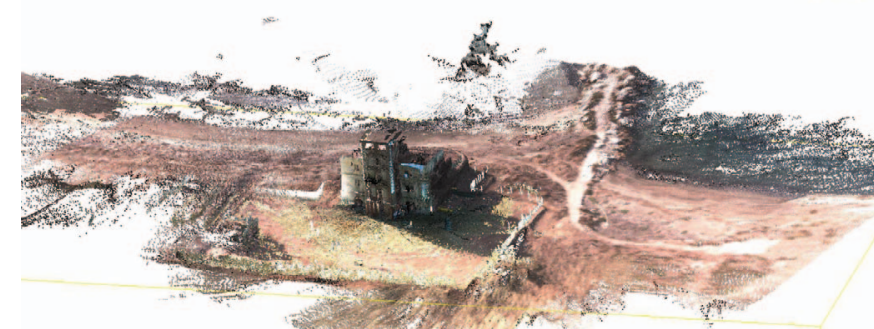

Figure 4 - Merged aerial and ground point clouds.

\section{AIR COMPONENT}

ICARUS follows a collaborative multi-stage approach for the aerial assessment of the disaster zone with multiple and complementary aerial systems (fixedwing and outdoors and indoors multirotors). However, only one of the aerial systems could participate in euRathlon according to the rules of the competition: an autonomous outdoor coaxial quadcopter. The platform is called LIFT VI (Figure 5) and has been developed by Eurecat-ASCAMM Technology Center to satisfy the needs of Search and Rescue operations.

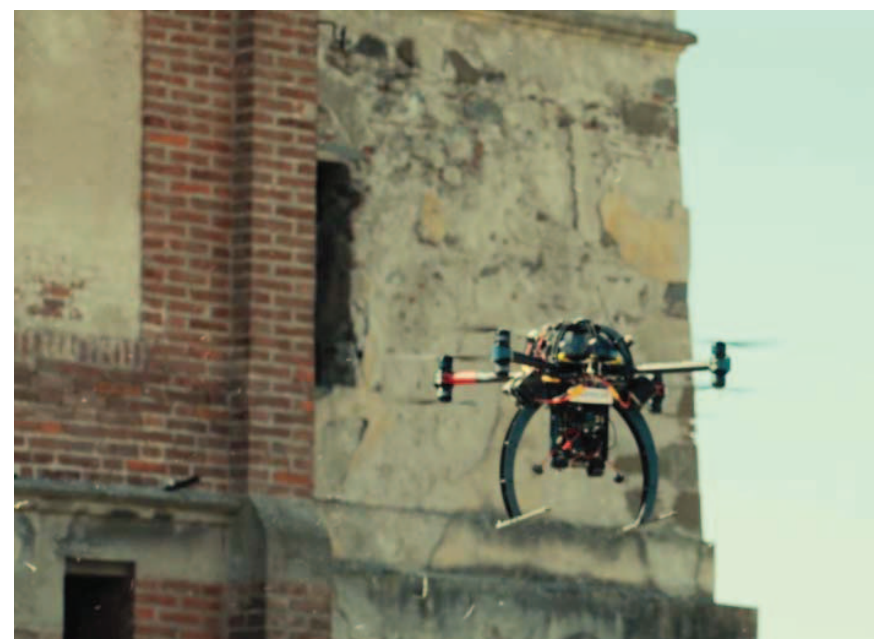

Figure 5 - ICARUS team UAV

LIFT IV has a 855 $\mathrm{mm}$ diameter and weights $4.3 \mathrm{Kg}$. The propulsion chain has been optimized to maximize the payload capacity (3.8 Kg), Figure 3- Eurecat MAV Copilot endurance (approx 40

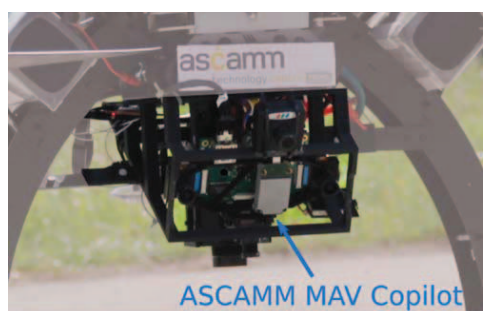

minutes) and wind resistance (up to $35 \mathrm{Km} / \mathrm{h}$ ). The system incorporates the Eurecat "Copilot" as illustrated in Figure 6 which is a payload designed specifically for Search and Rescue operations, integrating an onboard computer, a stereo vision-inertial sensor, a thermal imager (FLIR Tau2), and a mechanism to deliver survival kits to the victims. These features allow the automatic detection of victims, obstacle avoidance, fast mapping, 3D reconstruction and other added value functionality. All sensor data is automatically available at the C2I. The following image (Figure 7) shows the real time detection of the "Unblocked entrance" on the quadrotor. 


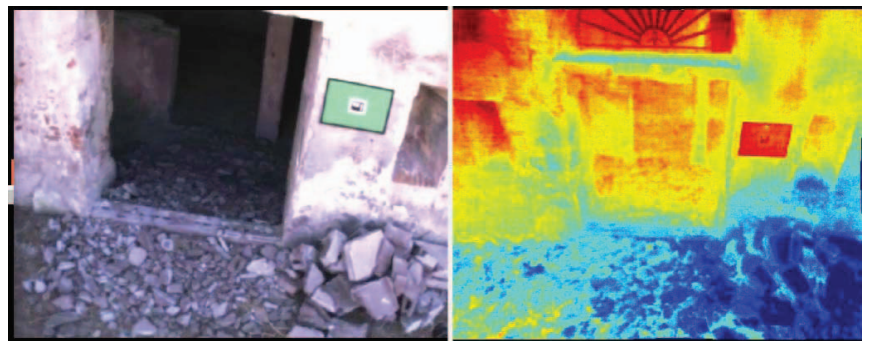

Figure 4 - Unblocked entrance detected on thermal and visual camera

\section{SEA COMPONENT}

The sea component was constituted by the MARES autonomous underwater vehicle (Figure 9) and the ROAZ autonomous surface robot (Figure 8). These two robotic systems provided complementary capabilities for the sea challenges and were used in cooperation.

The ROAZ unmanned surface vehicle is a $4 \mathrm{~m}$ long, $2 \mathrm{~m}$ wide catamaran type of vessel with electric propulsion capable of operating at sea. Its navigation system uses an on board fiber optic (FOG) INS system and a precise RTK GPS receiver thus allowing for accurate geo-referencing of sensory data [2].

On board processing capabilities allow not only for control, navigation and mission execution but also for sensor data processing such as autonomous detection of mission relevant events.

The vehicle as a set of surface mounted sensors (mounted on the bridge in Figure 1) such as visible spectrum and infrared cameras, a RADAR and a LIDAR sensor for close range environment modelling.

Underwater, it carries a single beam echo sounder, a multibeam and a side scan sonar. These sensors are used for seabed 3D modelling and bathymetry tasks.

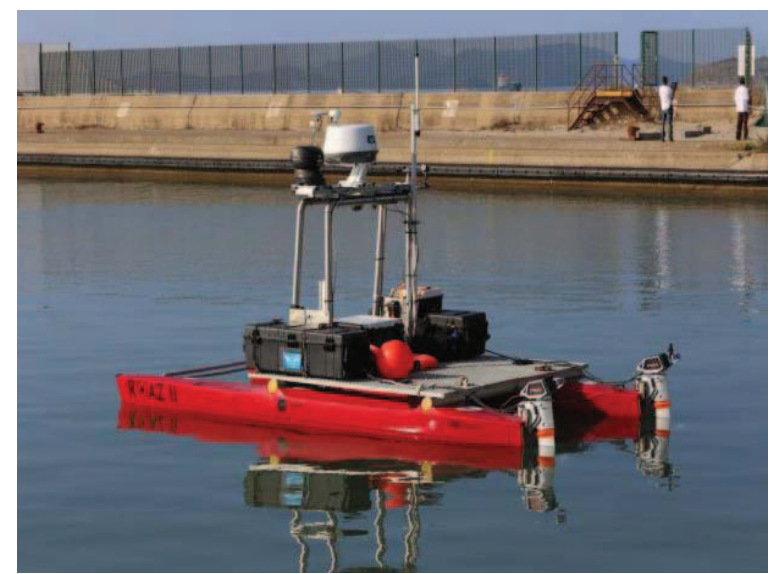

Figure 8 - ROAZ Unmanned Surface Vehicle

The MARES (Modular Autonomous Robot for Environment Sampling) autonomous underwater vehicle is a 1.5 meter long, $20 \mathrm{~cm}$ diameter vehicle capable of hovering operations with an $10 \mathrm{hr}$ of autonomy and diving up to $100 \mathrm{~m}$. This highly manoeuvrable vehicle has a digital video camera with on-board video processing. It also possesses a downward looking echosounder, a pressure cell and a inertial measurement unit, as basic navigation sensors. An acoustic LBL navigation system can also be used for geo-referenced operations.

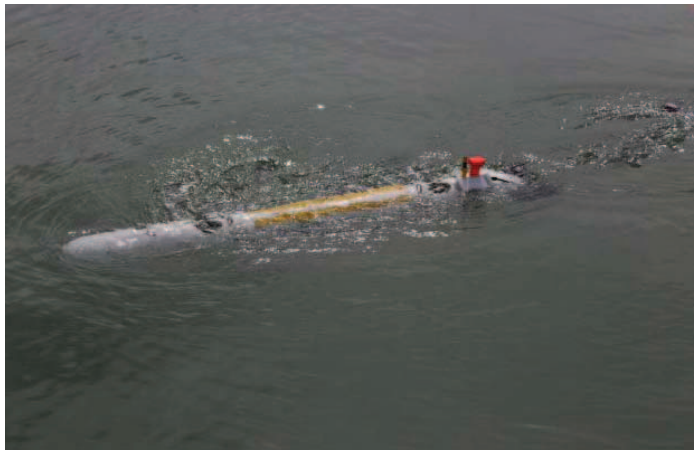

Figure 9 - MARES Autonomous Underwater Vehicle

ROAZ unmanned surface vehicle provided an initial survey of the area providing an initial bathymetry map along with possible points of interest to be searched by the AUV. This allowed for a relative fast coverage of the area and also was used as an input for the MARES AUV mission definition.

After the initial survey, MARES starts its mission diving already near one of the possible points of interest. With the on-board camera an underwater survey is performed and automatic detection of the relevant targets (human victim, plume leaks or piping structures in this case) is performed. All the logged images are georeferenced using information from the AUV navigation system, thus allowing the posterior location of the targets.

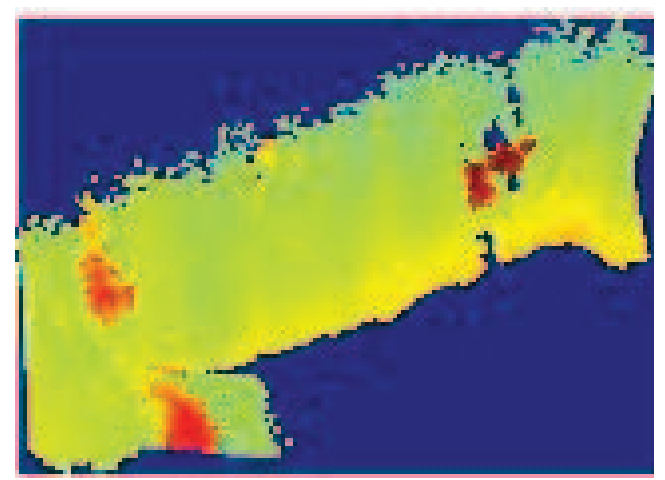

Figure 10 - Initial survey map made by ROAZ USV in one of the trials

In the previous figure (Figure 10) one example of one of the initial multibeam surveys is presented. The map shows the location of possible underwater structures of interest signaled in red.

On the following picture (Figure 11) an image taken by the AUV of one of the required targets (pipe entrance) is shown. The timestamp of all the data allowed for position correlation and location of the targets. 


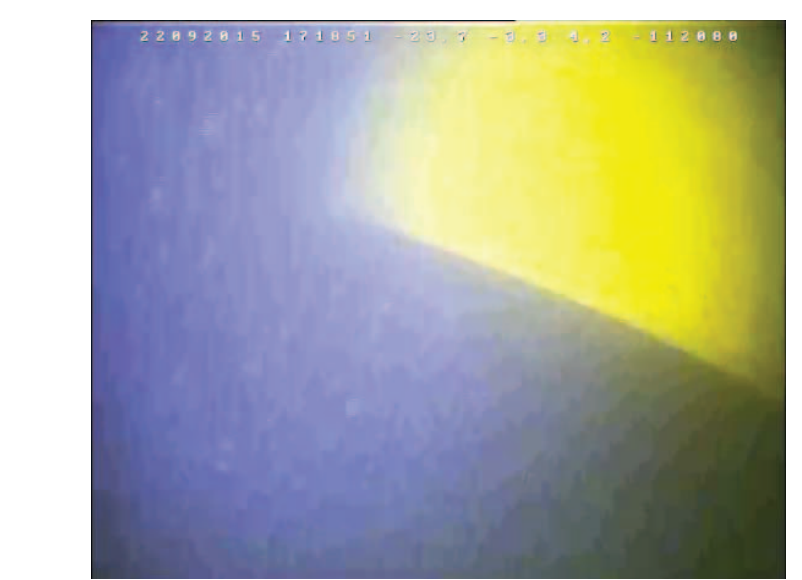
taken by MARES AUV

\section{LAND COMPONENT}

The land component of ICARUS team consists of two mobile platforms: heavy, outdoor oriented TEODOR and indoor oriented SUGV. During a mission the platforms cooperate to fully utilize strong points of both. TEODOR is equipped with a mapping system dedicated for gathering 3D data about the mission area. The system consists of rotating SICK LMS 500 scanner and LadyBug spherical camera. The data provide by the system are 3D point clouds and spherical images. Images are recorded during full robot operation while 3D data are gathered in stop-scan fashion.

During the competition TEODOR was responsible for transportation of SUGV and gathering data about the mission area. Transportation was done by using a custom made trailer that automatically disconnected from TEODOR when SUGV moved from it. The whole setup is shown in Figure 12. After release SUGV's main tasks is to perform inspection of indoor area and cope with and manipulation tasks required.

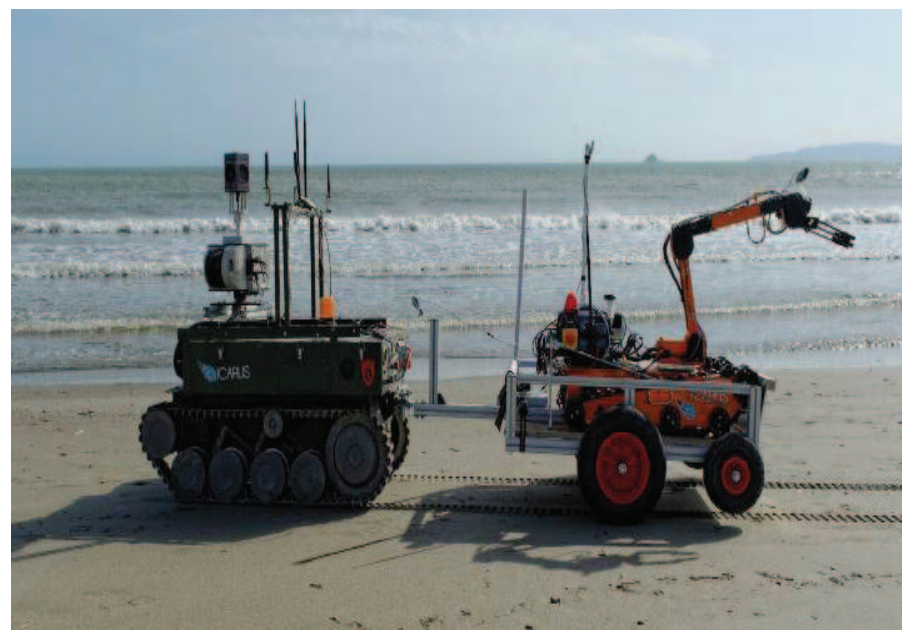

Figure 12 - ICARUS team land components: TEODOR, SUGV and trailer.

After releasing the smaller robot TEODOR's main tasks was to create a 3D map of the outdoor section of mission area. The map is being built online during robot operation. To achieve online operation single 3D scans have to be down-sampled (from $\sim 300.000$ to 3.000 points). An unfortunate side-effect of this approach is lower accuracy of 3D model. Because of this mapping framework can enhance the map in posts-processing, after the mission. Enhanced maps can achieve accuracy of up to $1 \mathrm{~cm}$ and have density similar to densities of single scans.

One of the most important side tasks in the competition was detection of OPI's in the mission area. With ground platforms an approach with 3 separate layers was used:

- Automatic detection - the cameras of the robots were connected to automatic detection software that recorded found OPI's in $\mathrm{kml}$ file.

- Operator detection - robot operators were equipped with tools for reporting and identifying OPI's. The tools allowed user to place the OPID on a georeferenced map and saved info in $\mathrm{kml}$ file

- Post-Processing detection - final layers of detection was done in post-processing by analyzing recorded LadyBug stream for potential omitted OPI's. This approach proved to be especially successful for OPIs that were in places with limited visibility.

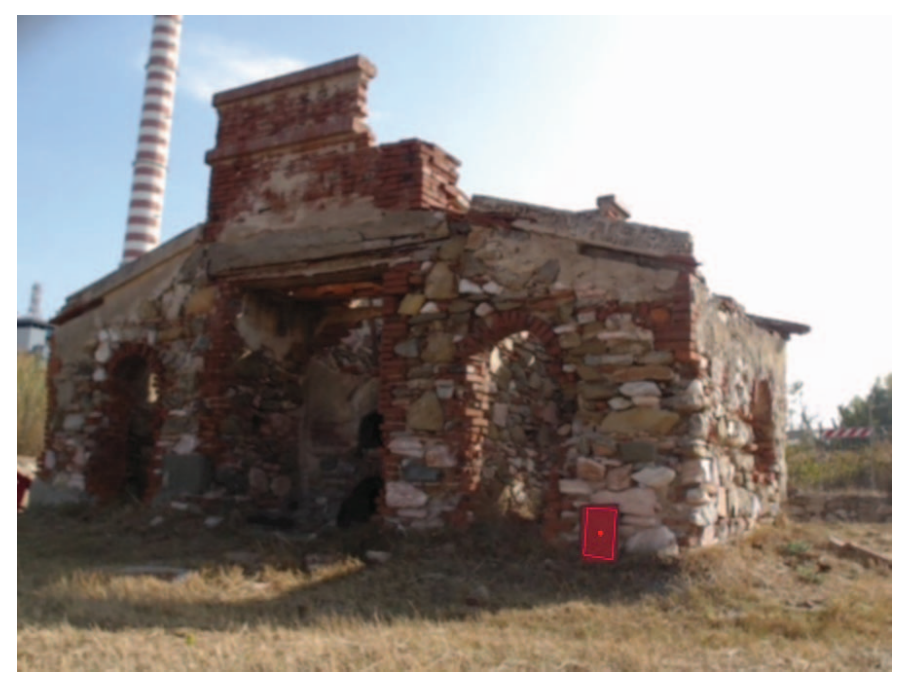

Figure 13 - Example of automatic OPI detection in image.

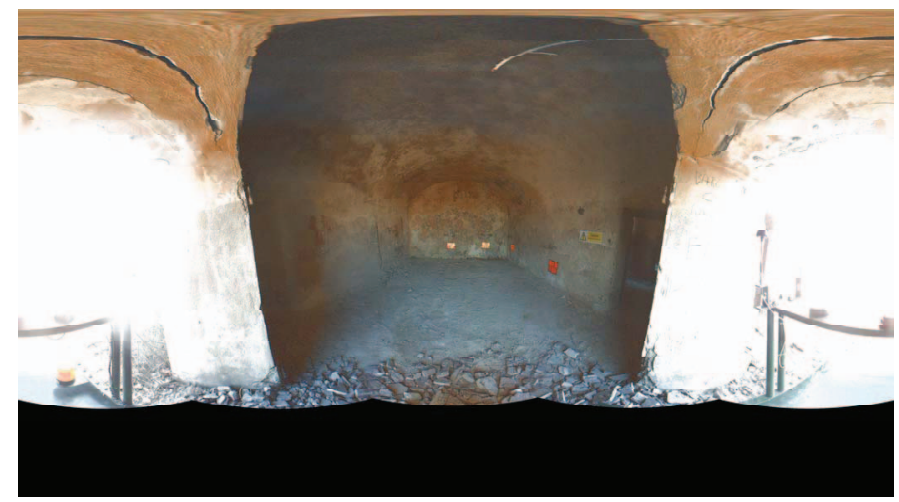

Figure 14 - Spherical image from Ladybug camera 
The main problem the ground platforms faced was communication. Both platforms used a $2.4 \mathrm{MHz}$ WI-Fi connection. This significantly lowered the reliability of the connection for higher ranges and indoors. Use of repeaters did not significantly improve the situation. Major improvement was noticed after changing part of robots antennas to such that use reflections instead of direct signal.

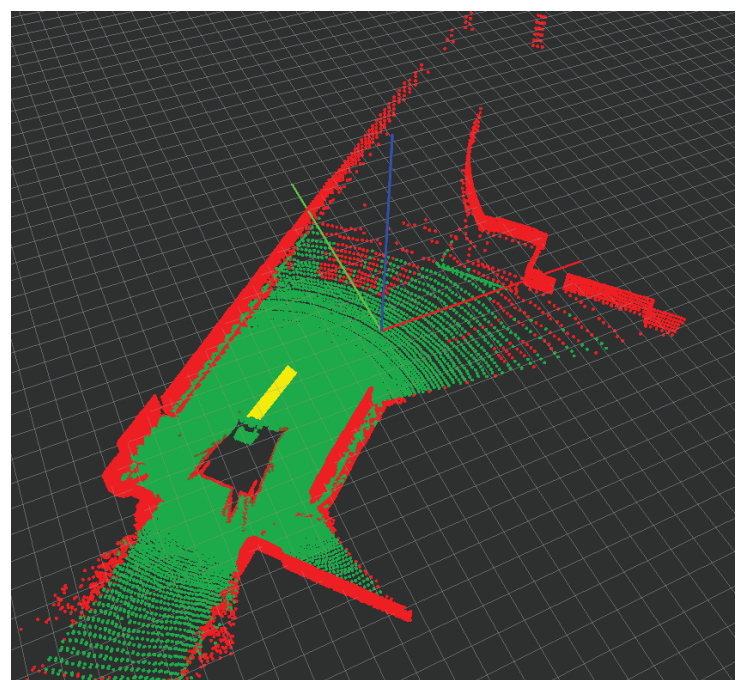

Figura 15 - Traversability map generated based on single scan; red - non-traversable, green traversable

TEODOR platform is able to operate in semiautonomous modes during mapping. The approach used is based on $3 \mathrm{D}$ point cloud data. Path to new scanning position is computed based on the last scan. In that mode robot travels about $2-3$ meters between scans. This distance is one of key parameters. Operator is able to define waypoints in latest local scan. During autonomous operation the system goes through following steps in a loop:

- Scan of environment - Laser scanner needs to perform full revolution. It takes about 10 seconds.

- Point cloud registration - Latest scan is localized and matched to previous scans. After this step robot is localized, and consistent map of environment is updated.

- Scan cropping, subsampling and normal vector computation - In this step the scan is cropped to remove points which are not important (higher than robot) for traversability reasoning. Point cloud is also down-sampled to achieve uniform density, which is important for proper normal vector computation.

- Scan classification - in this step points in the cloud are classified into two classes: traversable and non-traversable. Traversable points are those which lay on ground. Classifier uses data provided by normal vectors.

- Non traversable areas are projected on XOY plane
- 3 step - 2D inflation of map - 2D inflation (dilation with circular kernel with given radius) allows to simplify robot to a point in next steps. Added next inflation level with smaller weight prevents approaching obstacles.

- Generation of trajectories - Control space of the robot is discretized and constrained with minimal and maximum angular and linear velocity, and maximum angle.

- Trajectories simulation - For every generated trajectory $\mathrm{R}\left(\mathrm{v} \_\mathrm{a}, \mathrm{v} \_\mathrm{l}\right)$ in control space a trajectory is generated in output space $\mathrm{R}(\mathrm{x}, \mathrm{y}, \mathrm{fi})$.

- Trajectory are projected on 2D map and weighted.

Trajectory weight is sum of map cells' weights which trajectory contains. Also trajectories get penalty points if angular velocity is high. Additional penalty points are given if end of trajectory does not point to the goal.

- Trajectory with lowest weight is send to robot controller, if obtained weight is lower than threshold.

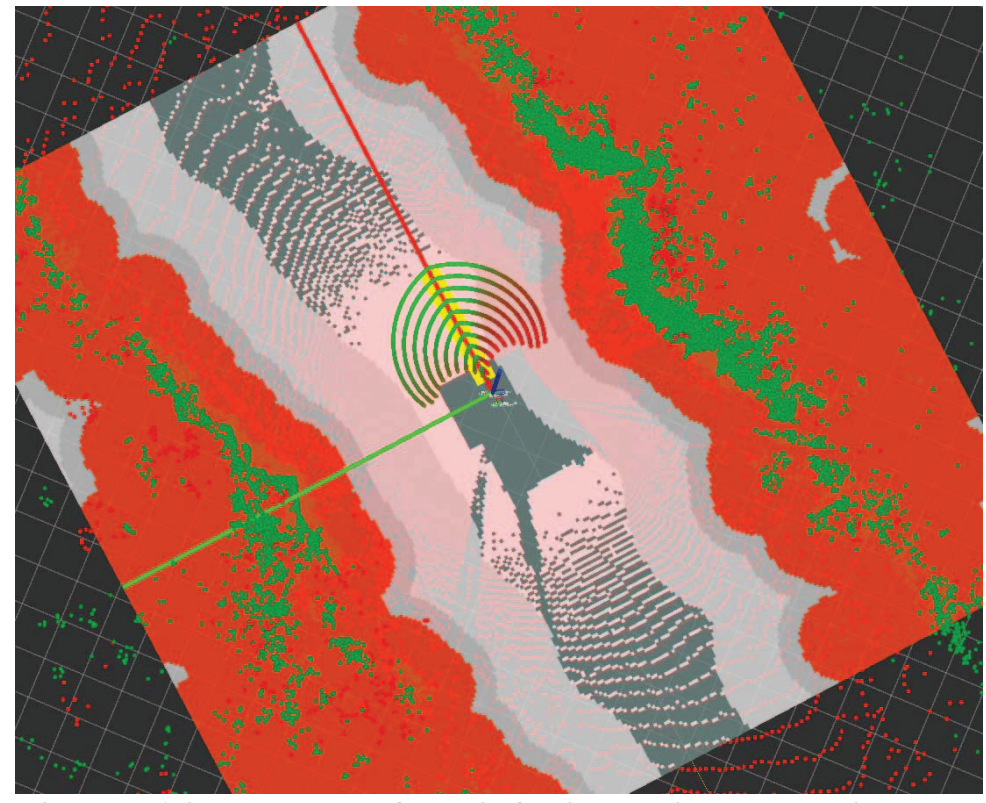

Figure 16 - Map after inflation with potential trajectories.

\section{CONCLUSIONS}

The major issue for successful implementation of ground mobile vehicles is communication. Lack of connection with robot leads to problems even in autonomous mode as operator has no possibility to oversee robot operation and intervene in critical situations.

Use of 3D laser and 360 cameras provides a major improvement in operators understanding of the scene. Even low quality images of the whole surrounding areas provide more information about general robot situation than normal quality classical cameras. 
In situations when connection with the robot has a high delay $3 \mathrm{D}$ point clouds are a good alternative for cameras. Lower refresh rate $(0.1 \mathrm{HZ}$ in comparison with $30 \mathrm{HZ}$ of standard camera) requires lower bandwidth and detailed geometrical information about immediate surroundings allow to perform semi-autonomous operation.

Detailed 3D models and spherical images gathered during platform operation provide a major improvement to global operational picture. Level of detail provided by the data makes them valuable for mission planning, evaluation and possible repetition.

\section{ACKNOWLEDGEMENTS}

The research leading to these results has received funding from the European Union Seventh Framework Programme (FP7/2007-2013) under grant agreement $n^{\circ}$ 285417 - ICARUS Project, and also from the ERDF European Regional Development Fund through the COMPETE Programme (operational programme for competitiveness).

\section{REFERENCES}

[1] Mario Monteiro Marques, P. Dias, N. Santos, V. Lobo, R. Batista, D. Salgueiro, R. Ribeiro, J. Marques, A. Bernardino, M. Griné, M. Taiana, M. Nunes, E. Pereira, J. Morgado, A. Aguiar, M. Costa, J. Silva, A. Ferreira, J. Sousa, "Unmanned Aircraft Systems in Maritime Operations: Challenges addressed in the scope of the SEAGULL project," in MTS/IEEE OCEANS 2015, 2015, pp. 1-6.

[2] Mario Monteiro Marques, Alfredo Martins, Anibal Matos, Nuno Cruz, José Miguel Almeida, José Carlos Alves, Victor Lobo, Eduardo Silva, "REX14 - Robotic Exercises 2014 - Multi-robot field trials," in MTS/IEEE OCEANS 2015, Washington 2015, pp. 1-6

[3] D. Serrano, G. De Cubber, G. Leventakis, P. Chrobocinski, D. Moore, S. Govindaraj, "ICARUS and DARIUS approaches towards interoperability". IARP RISE Workshop, At Lisbon, Portugal. Proceedings of the NATO STO Lecture Series SCI-271

[4] D. Serrano (2015). "Command and Reporting Standards and Associated Development Tools for $\mathrm{UxS}^{\prime \prime}$.

[5] H. Balta, J, Bedkowski, S. Govindaraj , K. Majek , P. Musialik, D. Serrano, K. Alexis, R. Siegwart, G. De Cubber. "Combined aerial and terrestrial 3D mapping for improving the situational awareness in search and rescue operations". (submitted-under minor revision) in Journal of Field Robotics 2016 (Submitted). 